\title{
On the Universality of Low-energy String Model
}

\author{
Tekin Dereli and Yuri N. Obukhov* \\ Department of Physics, Middle East Technical University, 06531 Ankara, Turkey
}

\begin{abstract}
The low-energy (bosonic "heterotic") string theory is interpreted as a universal limit of the Kaluza-Klein reduction when the dimension of an internal space goes to infinity. We show that such an approach is helpful in obtaining classical solutions of the string model. As a particular application, we obtain new exact static solutions for the two-dimensional effective string model. They turn out to be in agreement with the generalized no-hair conjecture, in complete analogy with the four and higher dimensional Einstein theory of gravity.
\end{abstract}

PACS no.: 04.60.Kz; 04.20.Jb; 03.40.Nr

Typeset using REVTEX

*On leave from: Department of Theoretical Physics, Moscow State University, 117234 Moscow, Russia 


\section{INTRODUCTION}

In this paper we demonstrate that the low-energy string action in a spacetime $M^{d}$ of an arbitrary dimension $d$ arises naturally in the framework of the Kaluza-Klein reduction from an infinite-dimensional spacetime. Such a reduction is constructed as a limit of $n \rightarrow \infty$ for the dimension $n$ of the internal space. We show that this limit exists and is universal in the sense that it does not depend on $M^{d}$ : for any $d$ the reduced action always turns out to be the string model in $M^{d}$.

Although it is at the moment unclear to us, whether this universality property has a deep physical meaning, one can use this fact as a technical tool for the study of both the string theories in an arbitrary dimension and the very higher dimensional Kaluza-Klein theories, propagating the knowledge from one model to another.

As a first example we can mention a possibility of obtaining new exact solutions for the low-energy string models from the exact solutions of the higher dimensional Einstein field equations. This method works for a string theory in any $M^{d}$, and below we illustrate it for the case $d=2$. On the other hand, in the study of $d+n$-dimensional configurations

one can use the underlying $d$-dimensional string action as a leading approximation and the specific features of the higher dimensions will be taken into account perturbatively as corrections proportional to the powers of $1 / n$. For example, one can obtain in this way the position of horizons, temperature, entropy and other physical and geometrical parameters of the higher-dimensional black holes, grasping the essential features coming from the string action.

\section{KALUZA-KLEIN THEORY IN INFINITE DIMENSIONS}

Let us consider the Kaluza-Klein reduction of a $d+n$-dimensional manifold to the physical $d$-dimensional Riemannian spacetime $M^{d}$ with an $n$-dimensional internal space of constant curvature. Denote the components of the higher dimensional curvature two-form $\mathcal{R}^{A B}$ with 
respect to a local orthonormal frame $E_{A}$. The dual coframe one-forms are denoted $\vartheta^{A}$, and the indices run $A, B, \ldots=0,1, \ldots, d+n$. The general Kaluza-Klein decomposition of the metric reads:

$$
\stackrel{(d+n)}{g}=\stackrel{(d)}{g}+e^{-\frac{4}{n} \Phi \stackrel{(n)}{g}}
$$

where $\Phi$ is the Kaluza-Klein scalar field which depends only on the coordinates of $M^{d}$, and

$$
\begin{aligned}
& \stackrel{(d)}{g}=g_{\alpha \beta} \vartheta^{\alpha} \otimes \vartheta^{\beta}, \\
& \stackrel{(n)}{g}=g_{a b} \vartheta^{a} \otimes \vartheta^{b},
\end{aligned}
$$

describe, respectively, the metric of the physical spacetime $\left[\right.$ with $g_{\alpha \beta}=\operatorname{diag}(-1,1, \ldots, 1)$ as a $d$-dimensional Minkowski metric] and the internal space [with $g_{a b}=\delta_{a b}$ ] of a constant curvature $R^{a b}=-\lambda \vartheta^{a} \wedge \vartheta^{b}$. The constant $\lambda=+1$ for an $n$-sphere of a unit radius, $\lambda=0$ for flat space (e.g., hyperplane, cylinder or $n$-torus), and $\lambda=-1$ for a hyperbolic space. The (local frame) indices clearly run: $\alpha, \beta, \ldots=0,1, \ldots, d-1$, and $a, b, \ldots=1, \ldots, n$.

Consider now, for concreteness, the Einstein-Maxwell-Klein-Gordon theory (with a cosmological term) in $d+n$ dimensions. The Lagrangian $(d+n)$-form reads

$$
L=-\frac{1}{2} \mathcal{R}^{A B} \wedge \eta_{A B}-\frac{1}{2} F \wedge \# F-\frac{1}{2} d \phi \wedge \# d \phi-\Lambda \eta
$$

Here $F=d A$ is the Maxwell field strength two-form and $\phi$ is the scalar field. For simplicity, we limit ourselves to the case of one massless real scalar field, however the whole scheme works in the same way also for an arbitrary multiplet of fields with different masses, as well as for the other types of matter (say, for fluids). We are using the general notations and conventions of [7]. In particular, the Trautman's $\eta$-basis of exterior forms is defined by the Hodge duals of the products of coframe one-forms $\vartheta^{A}$ : given the volume $(d+n)$-form $\eta$, one has $\left.\left.\eta_{A}=\# \vartheta_{A}=E_{A}\right\rfloor \eta, \eta_{A B}=\#\left(\vartheta_{A} \wedge \vartheta_{B}\right)=E_{A}\right\rfloor \eta_{B}$, etc. Same notation is used for the lower-dimensional counterparts in $M^{d}$.

Assuming that the matter fields (Maxwell and Klein-Gordon, in our present case) are independent of the internal space coordinates, we straightforwardly obtain from (2.4) a dimensionally reduced Lagrangian $d$-form: 


$$
\begin{aligned}
L=e^{-2 \Phi}(- & \frac{1}{2} R^{\alpha \beta} \wedge \eta_{\alpha \beta}+2 \frac{n-1}{n} d \Phi \wedge * d \Phi+\frac{1}{2} \stackrel{(n)}{R} e^{\frac{4}{n} \Phi} \eta \\
& \left.-\frac{1}{2} F \wedge * F-\frac{1}{2} d \phi \wedge * d \phi-\Lambda \eta\right) .
\end{aligned}
$$

Here: $\stackrel{(n)}{R}=\lambda n(n-1)$ is the curvature scalar of the internal space, and from now on $\eta$ denotes the volume $d$-form and $*$ is the $d$-dimensional Hodge operator on $M^{d}$.

Now we can immediately see that the formal limit $n \rightarrow \infty$ exists and it yields exactly the low-energy string model in an arbitrary dimension $d$ :

$$
L=\frac{1}{2} e^{-2 \Phi}\left(-R^{\alpha \beta} \wedge \eta_{\alpha \beta}+4 d \Phi \wedge * d \Phi+c \eta-F \wedge * F-d \phi \wedge * d \phi\right)
$$

The Kaluza-Klein field $\Phi$ becomes an effective dilaton with the correct kinetic term, whereas

the constant $c$ comes as a combination from $\Lambda$ and $\stackrel{(n)}{R}$ terms. In the simplest case for a compactification on a torus, $M^{d} \times T^{n}$, the latter contribution is absent completely. When the internal space has a nontrivial curvature, there is a subtlety though: the naive limit will yield a formally infinite result because $\stackrel{(n)}{R} \sim n^{2}$ for large $n$. However one can easily cure this by assuming that the constant $\lambda \sim 1 / n^{2}$ which is always possible to arrange with the help of a simple rescaling of the local coordinates. We will always assume this "regularization" to be performed before taking the limit.

It is worthwhile to note that the limit $n \rightarrow \infty$ effectively provides a complete decoupling of the physical and internal spaces, leaving us exactly on $M^{d}$, since the second term in the Kaluza-Klein line-element (2.1) is always trivial in that limit.

\section{SOLUTIONS OF A TWO-DIMENSIONAL STRING THEORY FROM HIGHER DIMENSIONS}

In order to demonstrate how one can use the $n \rightarrow \infty$ limit, we will obtain a family of new exact solutions of a two-dimensional string model from the Kaluza-Klein solutions.

¿From now on, let us put $d=2$ and consider the case of positive $\lambda$. The Kaluza-Klein reduced Lagrangian (2.5) then describes the general $2+n$-dimensional spherically symmetric 
configurations of the metric-matter coupled system.

Till now analytic solutions of the Einstein-Maxwell-Klein-Gordon field system with a non-trivial cosmological constant are unknown either in four or in higher dimensions. However, for the vanishing $\Lambda$ the general solution is available for an arbitrary dimension [11]. [It directly generalises the solutions [1 6, 10], and is most conveniently obtained with the help of the effective two-dimensional Poincaré gauge theory [8,94]. In our notation, this $2+n$-dimensional spherically symmetric solution reads:

$$
g=-f d t^{2}+\frac{q h^{-\left(\frac{n-2}{n-1}\right)}}{\lambda(n-1)^{2}} d r^{2}+h^{\frac{1}{n-1}} d \Omega_{\lambda}^{2},
$$

where $d \Omega_{\lambda}^{2}$ is the line element on the $n$-dimensional space of constant curvature $\lambda$, and the functions $f=f(r), q=q(r), h=h(r)$ are given by

$$
f=\frac{\left(x^{2}-1\right)^{2}}{\mathcal{D}^{2}}, \quad q=\mathcal{D}^{2}, \quad h=\mathcal{D}^{2} r^{2}
$$

Here the function

$$
\mathcal{D}(x):=\frac{k^{2}(x+1)^{2 \mu}-(x-1)^{2 \mu}}{\left(x^{2}-1\right)^{\mu-1}}
$$

depends on $r$ via the auxiliary variable

$$
x:=-\frac{M}{r}
$$

and $M, k^{2}$ and $\mu$ are arbitrary integration constants.

The dilaton $\Phi$, scalar $\phi$ and the Maxwell field strength 2-form $F$ are, respectively:

$$
\begin{aligned}
\Phi & =-\frac{1}{4}\left(\frac{n}{n-1}\right) \log h, \\
\phi & =\sqrt{\left(1-\mu^{2}\right)\left(\frac{n}{n-1}\right)} \log \left|\frac{x-1}{x+1}\right|, \\
F & =d A=4 M \mu \sqrt{k^{2}\left(\frac{n}{n-1}\right)} \frac{x^{2}-1}{h} d t \wedge d r,
\end{aligned}
$$

For the case when $k^{2} \neq 1$, the electromagnetic potential, $A=A_{0} d t$, is obtained form (3.7) in the form: 


$$
A_{0}=\sqrt{k^{2}\left(\frac{n}{n-1}\right)} \frac{1}{1-k^{2}} \frac{(x+1)^{2 \mu}-(x-1)^{2 \mu}}{k^{2}(x+1)^{2 \mu}-(x-1)^{2 \mu}},
$$

When $k^{2}=1$, we have a different expression,

$$
A_{0}=\sqrt{\left(\frac{n}{n-1}\right)} \frac{(x+1)^{2 \mu}}{(x+1)^{2 \mu}-(x-1)^{2 \mu}} .
$$

The difference between the two cases (3.8) and (3.9) is revealed when we analyse the configurations with the vanishing scalar field which arise for $\mu= \pm 1$. Then it is easy to see that the parameter $M$ is proportional to the total mass of a source, whereas $k^{2}$ is related to the charge $Q^{2}$ of a solution. The family (3.1)-(3.3) embraces all possible spherically symmetric charged configurations: The particular case $k^{2}=1$ yields the (higher-dimensional generalisation of) the Bertotti-Robinson solution [16], whereas for $k^{2} \neq 1$ one has a (cf. Tangherlini [15]) Reissner-Nordstrom type solution.

Taking the limit $n \rightarrow \infty$ is straightforward. As we mentioned above, one must only be careful about the formally infinite limit of the curvature of internal space. Taking this into account, we immediately obtain from Kaluza-Klein solution (3.1) and (3.5)-(3.7) the exact solution of the two-dimensional string model by putting $n \rightarrow \infty$ :

$$
\begin{aligned}
g & =-f d t^{2}+\frac{1}{c r^{2}} d r^{2}, \\
\Phi & =-\frac{1}{4} \log h, \\
\phi & =\sqrt{\left(1-\mu^{2}\right)} \log \left|\frac{x-1}{x+1}\right|, \\
F & =d A=4 M \mu k \frac{x^{2}-1}{h} d t \wedge d r .
\end{aligned}
$$

One can prove directly that (3.10)-(3.13) satisfy the field equations for the effective lowenergy string Lagrangian (2.6).

This new solution is generalising the uncharged [12] and charged [13, 14] black holes for the case when a massless scalar field is present.

In two dimensions, the curvature two-form has only one component, and its invariant description is given by the curvature scalar, $\left.\left.R=e_{\alpha}\right\rfloor e_{\beta}\right\rfloor R^{\alpha \beta}$. For the metric (3.10), one finds: 


$$
R=2 \mu c\left\{1-\frac{32 \mu k^{2} x^{2}}{\mathcal{D}^{2}}+\frac{4(1-\mu) x^{2}}{\left(x^{2}-1\right)^{2}}-\left(x^{2}+1\right) \frac{k^{2}(x+1)^{2 \mu-2}-(x-1)^{2 \mu-2}}{k^{2}(x+1)^{2 \mu}-(x-1)^{2 \mu}}\right\} .
$$

It is interesting to note that the solution with $\mu=0$ describes a flat spacetime with nontrivial dilaton and scalar field configurations. Solutions with $\mu>0$ and $\mu<0$ are related by the coordinate transformation $x \rightarrow-x$. Consequently, we can limit our attention to the case of $\mu>0$.

As wee see, for $k^{2} \neq 1$ the spacetime has three asymptotically flat regions which are obtained in the limit of $x \rightarrow 0(r \rightarrow \infty)$, and in the limit of $x \rightarrow \pm \infty(r \rightarrow \mp 0)$. The matter fields, Maxwell $F$ and scalar $\phi$, vanish in these regions. When $k^{2}=1$, the spacetime is not asymptoically flat. Instead, the curvature and the Maxwell field are approaching constant values, $R \rightarrow-2 c$ and $F \rightarrow-\sqrt{c} \eta$ (independent of $\mu$ ), in the above asymptotic regions.

For $\mu \neq 0$, the curvature (3.14) displays several singular points in a spacetime. Namely, there are singularities located at the roots of the function $\left[k^{2}(x+1)^{2 \mu}-(x-1)^{2 \mu}\right]$, that is at $x=\left(1 \mp k^{1 / \mu}\right) /\left(1 \pm k^{1 / \mu}\right)$, and at the points $x=1$ and $x=-1$. Comparing this with the matter field configurations (3.12)-(3.13), we find that these singularities of the curvature are naturally coming from the blowing up of the Maxwell field at $x=\left(1 \mp k^{1 / \mu}\right) /\left(1 \pm k^{1 / \mu}\right)$ and from the divergences of the scalar field at $x= \pm 1$.

The cases $\mu= \pm 1$ are special in the sense that the scalar field (3.12) vanishes then. Let us consider this case in more detail because it is closely related to the Reissner-Nordstrom solution in four dimensions. For $\mu=1$ the curvature (3.14) simplifies to

$$
R=2 c\left\{1-\frac{\left(k^{2}-1\right)\left(x^{2}+1\right)}{k^{2}(x+1)^{2}-(x-1)^{2}}-\frac{32 k^{2} x^{2}}{\left[k^{2}(x+1)^{2}-(x-1)^{2}\right]^{2}}\right\} .
$$

[For $\mu=-1$ one should interchange $(x+1)$ and $(x-1)$ in denominators.] Note that for $k^{2}=1$ we find $R=-2 c$, i.e. the spacetime is a hyperbolic two-dimensional de Sitter manifold, $R^{\alpha \beta}=c \vartheta^{\alpha} \wedge \vartheta^{\beta}$. The local coordinate transformation $\widetilde{x}=\frac{1}{2}\left(x+x^{-1}\right), \tilde{t}=t /(2 \sqrt{c})$ brings the metric (3.10) with $\mu=k^{2}=1$ to

$$
g=-c\left(\widetilde{x}^{2}-1\right) d \widetilde{t}^{2}+\frac{d \widetilde{x}^{2}}{c\left(\widetilde{x}^{2}-1\right)},
$$


This is the two-dimensional Bertotti-Robinson [16] type solution, see the relevant discussion [17] in the context of the effective string theory. Along with the curvature, Maxwell field is constant: $A=(\widetilde{x}+1) \sqrt{c} d \tilde{t}, F=-\sqrt{c} \eta$.

For $k^{2} \neq 1$, the function $\left[k^{2}(x+1)^{2}-(x-1)^{2}\right]$ is a quadratic polynomial which has two roots, reciprocal to each other,

$$
x_{1}=\frac{1-k}{1+k}, \quad x_{2}=\frac{1+k}{1-k} .
$$

These are the positions of the two electric charges which create the Maxwell field configuration (3.13).

Let us assume, for definiteness, that $k \geq 0$. Then for $k<1$ we have $0<x_{1} \leq 1 \leq x_{2}$, whereas for $k>1$, one finds $x_{2} \leq-1 \leq x_{1}<0$. The case $k=0$ describes a solution with the zero charge, then $x_{1}=x_{2}=1$, whereas $k=1$ is a special case of the Bertotti-Robinson type solution with constant curvature and electric field.

We can establish a direct correspondence with the Reissner-Nordstrom type solution by means of an appropriate coordinate transformations. Namely, let us introduce a new spatial coordinate $\rho$ via

$$
e^{\sqrt{c}\left(\rho+\rho_{0}\right)}:=\frac{k^{2}(x+1)^{2}-(x-1)^{2}}{4 x}
$$

and a new time coordinate $\tau=t /\left(1-k^{2}\right)$. Then the metric reads:

$$
g=-f(\rho) d \tau^{2}+f(\rho)^{-1} d \rho^{2}
$$

where

$$
f(\rho)=\left(1-e^{-\sqrt{c}\left(\rho+\rho_{0}\right)}\right)\left(1-k^{2} e^{-\sqrt{c}\left(\rho+\rho_{0}\right)}\right) .
$$

As one can straightforwardly see, the ratio of charge to mass of the solution (3.20) is equal $2 k /\left(1+k^{2}\right) \leq 1$. Note however, that although in 3.19$\left.)-3.20\right)$ one can formally take the limit of $k \rightarrow 1$, thus obtaining the extremal charged black hole, such a configuration is not related to the Bertotti-Robinson type solution (3.16) by any coordinate transformation. 
Turning to the general case $\mu^{2} \neq 1$, we find that the exact solutions (3.10)-(3.13) are no black holes. The points $x= \pm 1$, which descibe regular horizons for $\mu^{2}=1$, are true singularities now. This is completely analogous to the Einstein-Maxwell-Klein-Gordon solutions in four [1 [5] and in higher [6] dimensions. Thus, one can conlcude that the no-hair conjecture is supported by our results for the two-dimensionsional effective string theory.

\section{CONCLUSION}

We have shown that the low-energy action on an arbitrary spacetime $M^{d}$ can be treated as a Kaluza-Klein reduction in the infinite-dimensional manifold, $M^{d+n}, n \rightarrow \infty$.

We apply this observation to the study of exact solutions of the effective string models. For $d=2$ we obtain new static solutions (3.10)-(3.13) on the basis of the $n \rightarrow \infty$ limit. Recently, the model with a slightly more general than (2.6) action has been analysed in [13]. There, the exact analytic solutions were reported for the trivial scalar field $\phi=$ const, and some general arguments were put in favour of the no-hair conjecture in string theory. Our new solutions provide an explicit support of this conjecture.

The authors are grateful to TUBITAK for the support of this research. Y. N. O. is also grateful to the Department of Physics, Middle East Technical University, for hospitality. 


\section{REFERENCES}

[1] I.Z. Fisher, ZhETF 18 (1948) 636 (in Russian).

[2] A.I. Janis, E.T. Newman, and J. Winicour, Phys. Rev. Lett. 20 (1968) 878.

[3] R. Penney, Phys. Rev. 182 (1969) 1389.

[4] K.A. Bronnikov and V.N. Melnikov, in: "Problems of Gravity and Elementary Particle Theory" /Ed. K.P. Stanyukovich (Moscow: Energoatomizdat, 1974), 5, p. 80-91 (in Russian); N.V. Pavlov, K.A. Bronnikov, and M.A. Kovalchuk, in: "Problems of Gravity and Elementary Particle Theory" /Ed. K.P. Stanyukovich (Moscow: Energoatomizdat, 1975), 6, p. 147-158 (in Russian); K.A. Bronnikov, M.A. Kovalchuk, and N.V. Pavlov, in: "Problems of Gravity and Elementary Particle Theory" /Ed. K.P. Stanyukovich (Moscow: Energoatomizdat, 1976), 7, p. 119-131 (in Russian);

[5] A.G. Agnese and M. La Camera, Phys. Rev. D31 (1985) 1280.

[6] B.C. Xantopoulos and T.E. Dialynas, J. Math. Phys. 33 (1992) 1463.

[7] F.W. Hehl, J.D. McCrea, E.W. Mielke, and Y. Ne'eman, Phys. Rep. 258 (1995) 1.

[8] E.W. Mielke, F. Gronwald, Yu.N. Obukhov, R. Tresguerres, and F.W. Hehl, Phys. Rev. D48 (1993) 3648; Yu.N. Obukhov, Phys. Rev. D50 (1994) 5072; Yu.N. Obukhov, S.N. Solodukhin, and E.W. Mielke, Class. Quantum Grav. 11 (1994) 3069.

[9] Yu.N. Obukhov and F.W. Hehl, Black holes in two dimensions, in: Proceedings of 179.WE-Heraeus Seminar "Black Holes Theory and Observations", Bad Honnef, Germany, 18-20 Aug 1997, Eds. F.W. Hehl, C. Kiefer, and R.J.K. Metzler (Springer: Berlin) Lect. Notes Phys. 514 (1998) 289.

[10] T. Dereli, Phys. Lett. B161 (1985) 307; T. Dereli and A. Eriş, Doğa A9 (1985) 30; A. Eriş, J. Math. Phys. 18 (1976) 824.

[11] T.Dereli and Yu.N. Obukhov, Massless scalar fields and topological black holes, Preprint 
METU (November, 1998).

[12] G. Mandal, A.M. Sengupta, and S.R. Wadia, Mod. Phys. Lett. A6 (1991) 1685; E. Witten, Phys. Rev. D44 (1991) 314.

[13] O. Lechtenfeld and C.R. Nappi, Phys. Lett. B288 (1992) 72;

[14] M. D. McGuigan, C.R. Nappi, and S.A. Yost, Nucl. Phys. B375 (1992) 421; C.R. Nappi and A. Pasquinucci, Mod. Phys. Lett. A7 (1992) 3337.

[15] F.R. Tangherlini, Nuovo Cim. 27 (1963) 636.

[16] B. Bertotti, Phys. Rev. 116 (1959) 1331; I. Robinson, Bull. Acad. Pol. Sci. 7 (1959) 351.

[17] D.A. Lowe and A. Strominger, Phys. Rev. Lett. 73 (1994) 1468. 